\title{
Integrated Development of Communication Technology and Computer Technology
}

\author{
Daijiancong $^{1}$ \\ ${ }^{1}$ Tianjin University of Technology, College of Art, China
}

\begin{abstract}
As the information age comes, new technology has become an important backbone for global economic development. Economic globalization has made all countries and regions value the research and development of new technology. They will rely on new technologies systematically to enhance their economic status and improve the market competency. Therefore, the penetration and integration between different kinds of technologies has seen unprecedented boom. Among them, the development and combination between communication and computer technology is a typical example and has witnessed outstanding performance. At present, computer and telecom computerization equipment has become indispensable communication tools in social life. As a result, a new technology called network communication technology comes into being. This paper mainly discusses computer and communication technology based on existed facts. In the meantime, the integration between the two has been specifically interpreted. At last, it is expected that breakthroughs will be made in science and technology through effective cooperation methods.
\end{abstract}

\section{Introduction}

With the accelerated world economy and civilization integration and the in-depth reform and opening-up, good communication in study, life, and work will stimulate the overall science and development level of our country and the world. Information can meet the demands for sharing through computer technology. Communication technology can guarantee the message transfer and delivery between people. No matter it is communication technology or computer technology, it will facilitate the development of social civilization. The paper will discuss the technical concepts integrating these two technologies, to realize the function of sharing and treatment methods of informatization and show the convenience and safety the high technology brings to life.

\section{Technology related to electronic computer}

\subsection{System technology is the core technology of electronic computer}

In electronic computer technology, system technology is the core to maintain the completeness of computer system. It mainly includes structure, management, maintenance and application technology. Different system technology will have different functions and fulfill different tasks. The purpose of structure technology is to guarantee the process speed and replace humans in computation. It stands out in terms of performance. Management technology exists in the operation system and shall be completed through operational procedures. This technology will greatly enhance the computation level of the computers and come up with the calculation results very quickly. Therefore, much labor and costs will be reduced. The function of maintenance technology is to carry out allround examinations of the computer programs to locate and solve the troubles in time. Application technology covers a large range, which is mainly about the automation in design and the development and application of related software.

\subsection{Machine piece technology is the basis for electronic computer technology}

Machine piece technology is consisted of various electronic components. They have different functions and locations. But they all form an integrated device. Only a complete set of electronic equipment can give rise to complete computer technology. Therefore, when designing the computer, advanced electronic equipment and device will be introduced, so as to make sure the computer is safe and effective. In this way, the corresponding data analysis will be more scientific and make more sense. According to related researches, the complexity of computer programs mainly comes from the complicated logic. And the completeness and perfection of logic is the result of the coordination by advanced electronic equipment. Therefore, the exploration to computer requires good knowledge of 
advanced electronic equipment and corresponding research and development.

\subsection{Component technology is an indispensable part of computer technology}

The structure of computer is very complicated. It not only contains the electronic devices mentioned above, but also components. Similar to the features of electronic devices, each component has its own unique functions. Therefore, component technology is a significant part for computer technology. It will benefit the stable and fast operation of the computer.

\subsection{Installation technology is the key technology for computer}

As the electronic device and component technology continues to develop and upgrade, higher requirements have been posed on installation technology. The effective coordination between installation technology and electronic device and components is essential to the improvement of computer technology. ${ }^{[1]}$ From current condition, it can be said that computer tends to become smaller and smaller. Therefore, related departments should invest more in installation technology and try every effort to reach the international level.

\subsection{Integrated analysis technology is the development direction of computer technology}

Computer technology under knowledge economy is not only limited to the improvement in calculation speed and accuracy. Nowadays, what computer technology pursues is the stable operation of computer and the diversified application of computer technologies. Current technologies cannot meet the demands by economy and society. In order to meet the new requirements posed by the time and society, innovation and improvement in computer technology is very necessary. To find a comprehensive analysis technology to realize the transfer from data information to knowledge graphs and tables and to enable computer to voluntarily sort and summarize the data is of great importance. It will analyze the data based on the requirements of the clients so as to meet the demands by the users.

\section{Communication technology}

The current business range of communication industry in our country is very broad, while the communication methods is also diversified. But to certain extent, communication technology is about transferring and delivering all kinds of information through the network and realize the fast transfer of information in time and space. First of all, the main goal of communication technology is to effectively and quickly deliver messages in different forms. In other words, network serves a medium during the transferring process and communication is realized through the network; second, the continuous development of communication technology is mainly shown by the upgrade and perfection of data transmission technology and signal path. For data transmission technology, in recent years, technologies like the data exchange, and frequency and band transmission have been developed. The improvement of signal path is mainly about progress in optical fiber and satellite communication, etc.; lastly, digital communication technology and other advanced multi-media technologies has to some extent facilitated the research and development of communication technology.

\section{Integration of computer and communication technology}

\subsection{Development trend of communication technology and computer technology}

As a matter of fact, from the perspective of current science and technology level, the integration of communication technology and computer technology has gained outstanding achievements. It has become dominant part in the information time. The development of science and technology is infinite. In the future, the integration between communication and computer technology will only be strengthened. The corresponding results will be applied to more areas. Based on the exited integration achievements concerning these two technologies, there will be shocking findings, which will bring many surprises and exciting moments to people's life through the development of science and technology. In this way, in production and life, the utilization efficiency of resources will be improved and the competition will become fiercer, which will highlight the comprehensiveness of science and technology.

\subsection{Performance of the integration of communication and computer technology}

\subsubsection{Computer communication technology}

The realization of communication and computer technology is a technical innovation combining the features of these two technologies. Among them, computer communication technology is the major content of their integration. In computer communication technology, the computer terminals can be used to calculate the data and fulfill the tasks. The exchange and integration of information technology will then be realized. To be specific, printing technology, mobile telephone technology, cable technology and many others are examples of the application of computer communication technology. Through computer communication technology, the resources can be cleverly shared and analyzed to realize the obstacle-free circulation of information. Moreover, the computer communication technology has been applied to multiple computers for connection, so more complicated technical calculations and resource-sharing will be performed 
through such technology. All in all, computer communication technology can give the best play to the resources of each computer and make the process of information transmission and message delivery smooth.

\subsubsection{Information technology}

Under the time of big data, the society lays more emphasis on the collection, processing and analysis of information, the timely and accurate transfer of data, and reliable analysis by computer. These factors directly impact the social and economic development. Information technology is the technology to effectively process systematic and complex information. It involves many kinds of advanced science and technology to process the data. For example, it combines telecommunication technology to process the data in accordance with the user needs and presents the outcome in the form of pictures, texts, tables, and so on, to meet different demands in data by various users.

\subsubsection{Bluetooth technology}

Bluetooth technology is the effective form for current integration of communication and computer technology. ${ }^{[2]}$ It is also a technology that is widely applied. First of all, Bluetooth technology can realize short-distance transmission of documents, pictures, information data without any other costs. Through wireless connection, data transmission will be realized; besides, Bluetooth is convenient while the technical requirements are not high. The smaller the distance is, the higher the efficiency will be. It can realize the transmission between one user and the other, or between one user and some others, which benefit the users a lot. The main content of Bluetooth technology is shown in the following diagram:

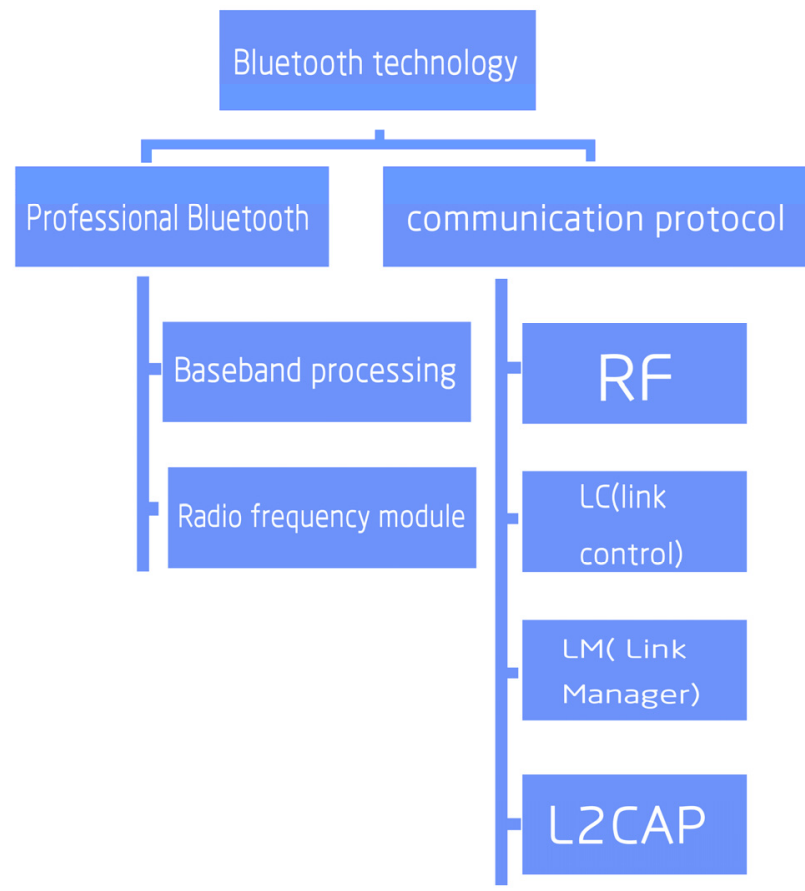

Among them, professional Bluetooth, the hardware structure of Bluetooth, is mainly for receiving the data. Communication protocol is about the safety and effectiveness of the system processor. Currently, Bluetooth technology has wide application. Many enterprises have come up with regulations and standards to regulate, update and manage this technology. From this, we can see that Bluetooth technology is development rapidly and the novel technical market centering on the integrated communication and computer technology is currently under exploration.

\subsubsection{Multi-media communication technology}

Multi-media communication technology is mainly about using computer technology to collect, summarize, categorize the information and to transfer the data. There are many technical approaches. These solve the following problems: the multi-media content should be sent in which format to occupy the least storage, how to achieve strong fault-tolerant capacity during transmission; how to fast transmit, and how to save the most resources. Multi-media communication technology has shortened the distance between computer and communication technology. The interaction of computers and distribution of communication has been connected to satisfy the requirements by some people.

\subsection{Advantages in technical integration of communication and computer technology}

\subsubsection{Obstacle-free space communication}

With the continuous progress of the time, the integration process of the world's economy and culture has been accelerated. People are having higher and higher requirements for communication beyond space. In order to realize this purpose, the integration between communication and computer technology can substantially realize the purpose of long-distance communication. The application of wireless computer network promotes the communication beyond time and space, through which good service can be provided using communication technology.

\subsubsection{Multimedia information support system}

Computer technology integrates traditional communication technology and provides more superior service system to help with the high-quality and non-stop transmission of data and information. During the process of transmission or delivery, in order to make people feel the dimensions of the data, data programing has been made to effectively deliver the information and data through voice, impact and texts. In this way, people's life quality can be leveled up. There are many examples for the application modern communication technology in daily life: to have an online conference in the company, long-distance education between students and teachers, and sending electronic contracts for the world trade 
business, and so on. There are also many inventions based on communication technology, for example, the popularization and application of Bluetooth, etc.

\section{Conclusion}

The economic time has brought opportunities for the integrated development of communication technology and computer technology. The outcome of information technology, computer communication technology and other technologies will be the main production force. The development and innovation in high technology industry requires continuous research on computer technology, communication technology and other modern scientific technologies.

\section{References}

1. W.B. Wan. Research on the role of computer technology in the promotion and development of communication technology,Digital technology and application,(2015)

2. L.Z.C.Liu. The integration of communication technology and computer technology under the market economy environment. Knowledge economic,(2015) 